02;09

\title{
Интерференция при туннельной ионизации электрона, связанного двумя короткодействующими потенциалами
}

\author{
(C) П.А. Головинский ${ }^{1,2}$, А.А. Дробышев ${ }^{1}$ \\ ${ }^{1}$ Воронежский государственный архитектурно-строительный университет, \\ Воронеж \\ ${ }^{2}$ Московский физико-технический институт (государственный \\ университет), Долгопрудный \\ E-mail: golovinski@bk.ru \\ Поступило в Редакцию 3 августа 2016 г. \\ Рассмотрена туннельная ионизация электрона, связанного двумя дельта- \\ потенциалами, под действием постоянного электрического поля. Получены \\ распределения плотности электронного тока для двух разных начальных состо- \\ яний. Исследована зависимость эмиссионного тока от ориентации потенциалов \\ относительно направления поля и расстояния между ними. Определены условия \\ проявления интерференционных эффектов.
}

DOI: $10.21883 /$ PJTF.2017.04.44304.16452

Метод потенциалов нулевого радиуса широко используется в атомной и ядерной физике, а также в физике конденсированных сред [1-3]. Важными приложениями метода стали описание нелинейной ионизации, генерации высоких гармоник в сильных лазерных полях [4-7] и простая модель туннельного эффекта в атомной микроскопии [8]. Известно аналитическое решение задачи о поляризации и туннельном отрыве электрона из короткодействующего потенциала атомарного отрицательного иона. Изучено также фоторазрушение отрицательных ионов в присутствии постоянного электрического поля разной конфигурации $[9,10]$. Используемый при этом единичный дельта-потенциал не описывает возможность делокализации электрона между несколькими областями притяжения, что на самом деле характерно для молекулярных и твердотельных систем.

Для задачи туннельной ионизации нейтральных молекул получило развитие обобщение метода сшивания асимптотик волновых функций 
в параболических координатах на случай сферически несимметричных состояний [11-13]. Однако и здесь теория пока ограничивается рассмотрением локализованных состояний отдельных молекулярных орбиталей. Представление о локализованных состояниях, не учитывающее их возможную интерференцию, лежит в основе описания туннельных эффектов в наноструктурах $[14,15]$. В то же время теоретически показано, что делокализация существенно влияет на рассеяние аттосекундных лазерных импульсов двухатомными молекулярными анионами [16]. Выяснение возможной роли делокализации в формировании электронного тока при туннельной ионизации требует специального рассмотрения.

Задача о движении частицы в поле нескольких короткодействующих потенциалов без внешнего поля допускает проведение полного аналитического исследования [17]. При относительной близости потенциальных ям волновые функции в них перекрываются, и образуется общее протяженное состояние. Кроме того, такие системы обладают специфическим откликом на внешнее поле [18]. Нами рассмотрена картина интерференции электронных волн, образующихся при туннельном отрыве электрона из двухъямного потенциала, при разной ориентации поля и потенциала.

Задачу о движении частицы в поле нескольких дельта-потенциалов можно сформулировать на языке граничных условий, накладываемых на волновую функцию в точках расположения потенциалов. Для одиночного $s$-состояния с $l=0$ волновая функция $\psi \sim r^{-1} \exp (-\alpha r)$, и граничное условие принимает вид [2]

$$
\left.\frac{d \ln (r \psi)}{d r}\right|_{r=0}=-\alpha
$$

где $\alpha=\sqrt{-2 E_{0}}, E_{0}-$ энергия связи. Мы пользуемся атомной системой единиц, в которой $|e|=m=\hbar=1$.

Для нескольких потенциалов их действие задается суммой одиночных потенциалов. Состояние частицы в поле двух одинаковых центров притяжения и внешнем электрическом поле с напряженностью $F$ описывается стационарным уравнением Шредингера

$$
\left(\varepsilon+\frac{1}{2} \nabla^{2}+F z\right) \psi(\mathbf{r})=\left(V_{1}\left(\mathbf{r}-\mathbf{r}_{1}\right)+V_{2}\left(\mathbf{r}-\mathbf{r}_{2}\right)\right) \psi(\mathbf{r}),
$$

где каждый потенциал $[1,3]$

$$
V(\mathbf{r})=-\frac{2 \pi}{\alpha} \delta(\mathbf{r}) \frac{\partial}{\partial r} r
$$

Письма в ЖТФ, 2017, том 43, вып. 4 
Решение, как и в задаче без поля [19], ищется в виде суперпозиции функций Грина

$$
\psi(\mathbf{r})=A G\left(\mathbf{r}, \mathbf{r}_{1}, \varepsilon\right)+B G\left(\mathbf{r}, \mathbf{r}_{2}, \varepsilon\right),
$$

где $G\left(\mathbf{r}, \mathbf{r}_{j}, \varepsilon\right)$ - функции Грина с асимптотикой уходящей волны, удовлетворяющие уравнению (2) с заменой правой части на $\delta\left(\mathbf{r}-\mathbf{r}_{j}\right)$, $\mathbf{r}_{1}$ - радиус-вектор первого центра, $\mathbf{r}_{2}$ - радиус-вектор второго центра (рис. 1,a). Функция Грина частицы, движущейся под действием постоянной силы, выражается через функции Эйри $\operatorname{Ai}(u)$ и $\operatorname{Bi}(u)$ [8]:

$$
\begin{gathered}
G\left(\mathbf{r}, \mathbf{r}^{\prime}, \varepsilon\right)=\frac{1}{2\left|\mathbf{r}-\mathbf{r}^{\prime}\right|}\left(\mathrm{Ci}\left(\chi_{+}\right) \mathrm{Ai}^{\prime}\left(\chi_{-}\right)-\mathrm{Ci}^{\prime}\left(\chi_{+}\right) \operatorname{Ai}\left(\chi_{-}\right)\right), \\
\chi_{ \pm}=-(2 F)^{-2 / 3}\left(2 \varepsilon+\mathbf{F}\left(\mathbf{r}+\mathbf{r}^{\prime}\right) \pm F\left|\mathbf{r}-\mathbf{r}^{\prime}\right|\right) .
\end{gathered}
$$

Функция $\mathrm{Ci}(u)=\operatorname{Bi}(u)+i \operatorname{Ai}(u)$ имеет асимптотику уходящей волны при $u \rightarrow \infty$.

Учитывая граничные условия вида (1), получим систему уравнений для комплексной квазиэнергии $\varepsilon=\operatorname{Re} \varepsilon-i \Gamma / 2$ и коэффициентов $A$ и $B$ :

$$
\frac{1}{\rho_{j} \psi} \frac{\partial}{\partial \rho_{j}}\left(\rho_{j} \psi\right)_{\rho_{j}=0}=-\alpha,
$$

где $\boldsymbol{\rho}_{\boldsymbol{j}}=\mathbf{r}-\mathbf{r}_{j}, j=1,2$. Эту систему можно записать в виде [20]

$$
\begin{gathered}
\left(\begin{array}{cc}
b_{+} & G(-\mathbf{R} / 2, \mathbf{R} / 2, \varepsilon) \\
G(\mathbf{R} / 2,-\mathbf{R} / 2, \varepsilon) & b_{-}
\end{array}\right)\left(\begin{array}{l}
A \\
B
\end{array}\right)=0, \\
b_{ \pm}=\frac{\pi}{(2 F)^{1 / 3}}\left(\operatorname{Ai}^{\prime}\left(\xi_{ \pm}\right) \mathrm{Ci}^{\prime}\left(\xi_{ \pm}\right)-\xi_{ \pm} \operatorname{Ai}\left(\xi_{ \pm}\right) \operatorname{Ci}\left(\xi_{ \pm}\right)\right)+\alpha, \\
\xi_{ \pm}=-\frac{2 \varepsilon \pm F R \cos \theta}{(2 F)^{2 / 3}},
\end{gathered}
$$

где $\mathbf{r}_{1}=\mathbf{R} / 2, \mathbf{r}_{2}=-\mathbf{R} / 2, R=\left|\mathbf{r}_{1}-\mathbf{r}_{2}\right|$. Система уравнений (3) имеет два решения, определяющих две волновые функции и два значения энергии $\varepsilon_{ \pm}$соответствующих состояний с их ширинами, которые находятся из условия равенства детерминанта нулю.

Из системы уравнений (3) следует также связь коэффициентов $A$ и $B$ :

$$
\frac{A}{B}=-\left.\frac{2 \pi G(-\mathbf{R} / 2, \mathbf{R} / 2, \varepsilon)+b_{ \pm}}{2 \pi G(\mathbf{R} / 2,-\mathbf{R} / 2, \varepsilon)+b_{\mp}}\right|_{\varepsilon=\varepsilon_{ \pm}} .
$$

Письма в ЖТФ, 2017, том 43, вып. 4 

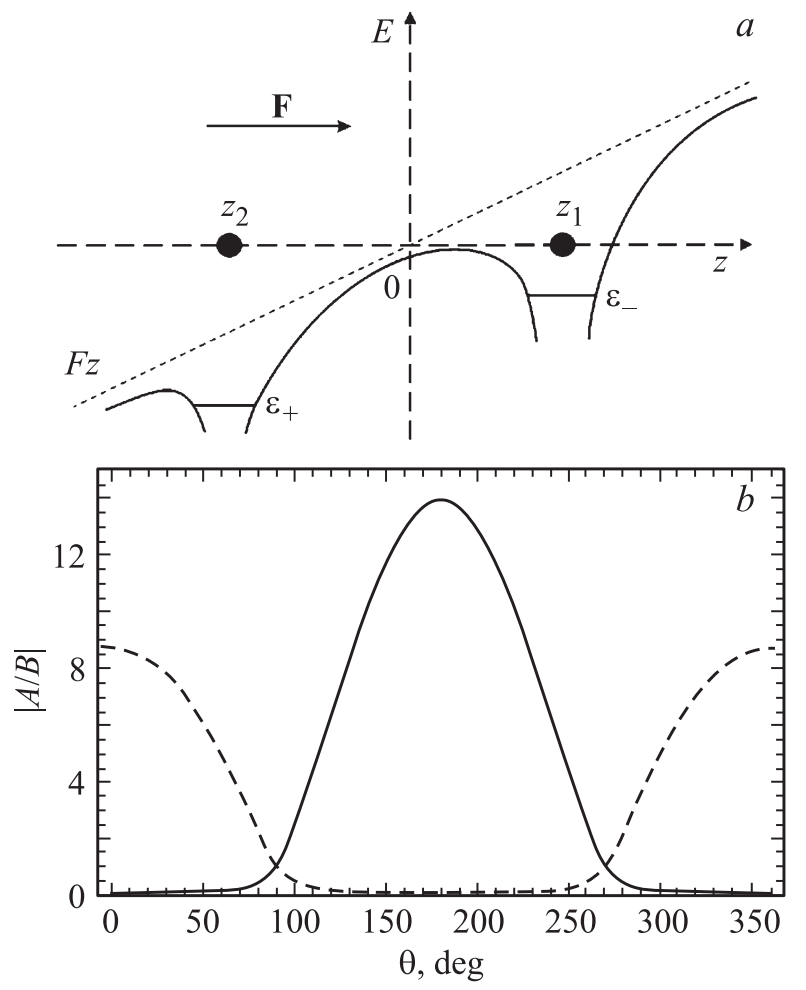

Рис. 1. Взаимное расположение центров $(a)$ и зависимость отношения коэффициентов $|A / B|$ от угла $\theta(b)$. Сплошная линия - основное состояние, пунктир — возбужденное.

Для слабого электрического поля $\left(F / \alpha^{3} \ll 1\right)$ сдвиги уровней и ширины вычислены ранее [18] и зависят от угла $\theta$ между линией, соединяющей центры, и направлением электрического поля.

Наблюдаемой величиной в процессе туннельной ионизации является плотность электронного тока, пересекающего плоскость, перпендикулярную оси $z$, задаваемой направлением электрического поля:

$$
j_{z}=\operatorname{Im}\left(\psi^{*} \frac{\partial \psi}{\partial z}\right) .
$$

Письма в ЖТФ, 2017, том 43, вып. 4 
Пространственное распределение электронного тока зависит от угла $\theta$ между осью, соединяющей центры, и направлением электрического поля, параметра $\alpha\left|\mathbf{r}_{1}-\mathbf{r}_{2}\right|$, характеризующего расстояние между центрами в сравнении с затуханием невозмущенной волновой функции, локализованной на отдельном центре, а также от параметра $F / \alpha^{3}$, задающего величину силы действия электрического поля по отношению к характерной силе, удерживающей частицу в потенциальной яме.

На рис. $1, b$ показан результат расчета отношения коэффициентов $|A / B|$ в зависимости от угла $\theta$ между $\mathbf{R}$ и $\mathbf{F}$ для значений параметров $E_{0}=1 \mathrm{eV}, F=10^{7} \mathrm{~V} / \mathrm{cm}, R=10$ a.u. Из представленных на рисунке зависимостей видно, что при $\theta=\pi / 2$ отношение коэффициентов в основном и возбужденном состояниях $|A / B|=1$, что соответствует ситуации осевой симметрии. При ориентации системы параллельно полю $(\theta=0)$ в основном состоянии $|A / B|=0.07$, т. е. электрон фактически локализован на втором центре. В возбужденном состоянии, при $\theta=0$, отношение коэффициентов $|A / B|=9$, что соответствует локализации электрона на первом центре.

Имеются два предельных случая ориентации двухъямного потенциала относительно поля: параллельно полю и перпендикулярно полю. При параллельной ориентации $(\theta=0)$ отношение коэффициентов $A / B$ зависит от напряженности поля и расстояния между ямами. В этом случае внешнее поле вызывает делокализацию исходных состояний. Вычисления показывают, что увеличение расстояния и напряженности поля приводит к уменьшению смешения состояний, и электрон локализуется на одном из центров. В основном состоянии электрон локализуется на втором центре и туннелирует практически без взаимодействия с первым центром, а распределение туннельного тока аналогично току от одного точечного источника (рис. $2, a$ ). В возбужденном состоянии электрон локализуется на первом центре и после туннелирования может рассеиваться на втором центре [20].

При ориентации двухъямного потенциала перпендикулярно полю $(\theta=\pi / 2)$ отношение коэффициентов $|A / B|=1$. Это означает, что волновая функция электрона является суперпозицией волновых функций в каждом из дельта-потенциалов, и электрон одинаково локализован на обоих центрах. В этом случае при ионизации электрона центры ведут себя как точечные когерентные источники (рис. 2, $b$ ). Внешнее поле в такой геометрии не вызывает делокализации исходных состояний.

На рис. 3 показан результат расчета поперечного распределения электронного тока при двух разных расстояниях между потенциалами.

Письма в ЖТФ, 2017, том 43, вып. 4 

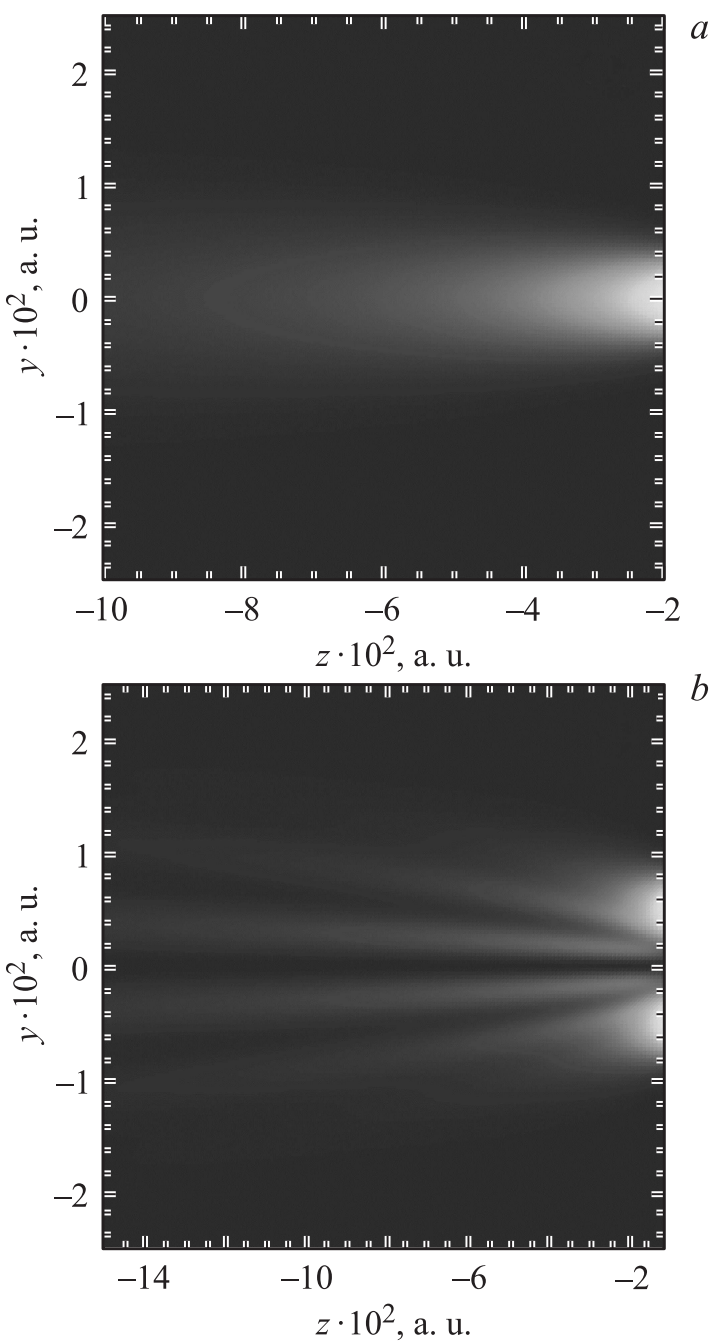

Рис. 2. Распределение электронного тока из основного состояния при значении угла $\theta=0(a)$ и $\theta=\pi / 2(b)$. Напряженность поля $F=10^{7} \mathrm{~V} / \mathrm{cm}, E_{0}=1 \mathrm{eV}$, $R=100$ a.u.

Письма в ЖТФ, 2017, том 43, вып. 4 


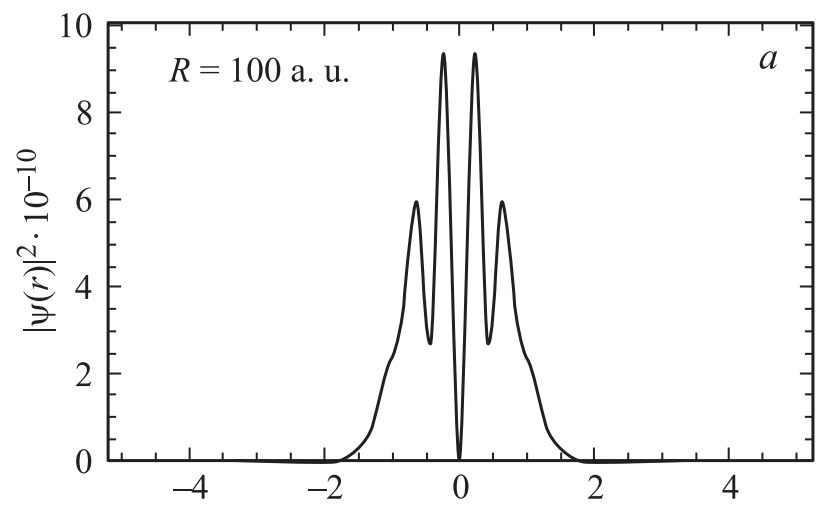

$y \cdot 10^{2}$, a. u.

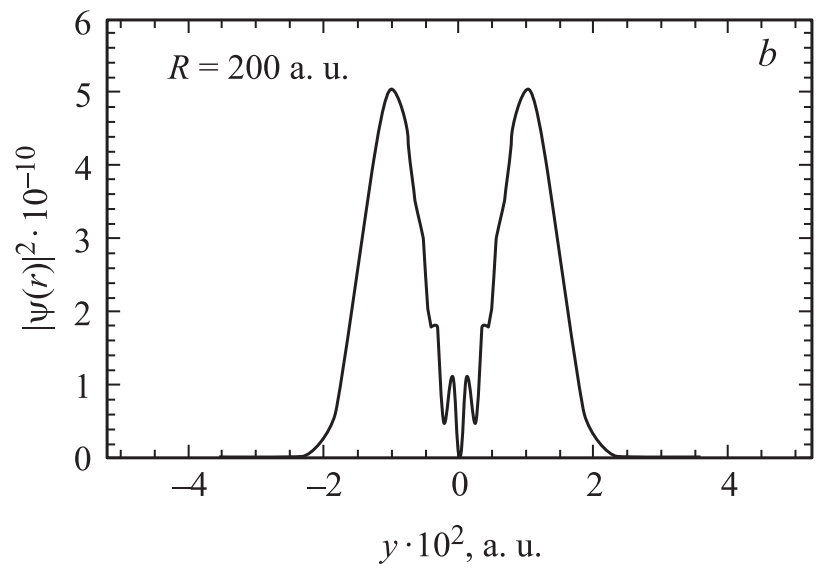

Рис. 3. Распределение электронного тока из основного состояния при разных значениях расстояния между потенциалами. Угол $\theta$ равен $\pi / 2$. Расстояние до экрана составляет 500 a.u., $E_{0}=1 \mathrm{eV}, F=10^{7} \mathrm{~V} / \mathrm{cm}$.

Сравнения представленных зависимостей демонстрирует, что при увеличении расстояния между центрами картина приближается к распределению тока от двух одинаковых независимых точечных источников.

Рассмотренное в работе явление интерференции электронных волн, образующихся при туннельной ионизации электрона из двухьямного

Письма в ЖТФ, 2017, том 43, вып. 4 
потенциала, существенно зависит от взаимной ориентации поля и потенциала, влияющей на степень локализации электрона возле разных центров. При ориентации двухъямной системы перпендикулярно полю распределение электронного тока эквивалентно распределению тока от двух одинаковых точечных когерентных источников. С ростом расстояния между центрами распределение приближается к картине, характерной для двух независимых источников. При наклонной ориентации потенциала, отличной от расположения перпендикулярно полю, делокализация исходных состояний увеличивается с ростом напряженности поля и расстояния между центрами.

Наблюдение описанных эффектов интерференции возможно в двухатомных молекулярных анионах [16]. Однако в таких экспериментах требуется решить дополнительные вопросы, связанные с выстраиванием оси молекулярного иона в заданном направлении и учетом или фиксацией его пространственного положения. В то же время современные технологии позволяют создавать наноэмиттеры из набора острых наноиголок [21,22], а также в виде нанопирамиды с вершиной из одного атома [23]. Близкое расположение таких наноразмерных источников будет обусловливать интерференционные проявления в токе эмиссии $[24,25]$. Это позволяет экспериментально проверить особенности пространственного распределения тока, предсказываемые предложенной моделью. Проведенные расчеты указывают также в целом на важность учета типа связи в молекулах при интерпретации результатов наблюдения туннельной ионизации электронов и необходимость проведения дополнительных исследований в этом направлении.

Работа выполнена в рамках ГЗ Министерства образования и науки РФ № 2014/19-2881.

\section{Список литературы}

[1] Демков Ю.Н., Островский В.Н. Метод потенциалов нулевого радиуса в атомной физике. Л.: ЛГУ, 1975. 240 с.

[2] Базь А.И., Зельдович Я.Б., Переломов А.М. Рассеяние, реакции и распады в нерелятивистской квантовой механике. М.: Наука, 1971. 544 с.

[3] Киржснии Д.А. Полевые методы теории многих частиц. М.: Либроком, 2010. $344 \mathrm{c}$.

[4] Frolov M.V., Manakov N.L., Starace A.F. // Phys. Rev. A. 2008. V. 78 (6). P. $063418(1-27)$.

Письма в ЖТФ, 2017, том 43, вып. 4 
[5] Frolov M.V., Flegel A.V., Manakov N.L., Starace A.F. // Phys. Rev. A. 2007. V. 75 (6). P. 063407 (1-14).

[6] Borzunov S.V., Frolov M.V., Ivanov M.Y. et al. // Phys. Rev. A. 2013. V. 88 (3). P. 033410 (1-18).

[7] Frolov M.V., Knyazeva D.V., Manakov N.L. et al. // Phys. Rev. A. 2014. V. 89 (6). P. 063419 (1-18).

[8] Donner B., Kleber M., Bracher C., Kreuzer H.J. // Am. J. Phys. 2005. V. 73 (8). P. $690-700$.

[9] Головинский П.А. // ЖЭТФ. 1997. Т. 112. В. 5. С. 1574-1583.

[10] Golovinski P.A., Drobyshev A.A. // Proc. SPIE. 2010. V. 7993. P. 799311 (1-9).

[11] Batishev P.A., Tolstikhin O.I., Morishita T. // Phys. Rev. A. 2010. V. 82. P. $023416(1-14)$.

[12] Jiang W.-Ch., Tolstikhin O.I., Peng L.Y., Gong Q. // Phys. Rev. A. 2012. V. 85. P. $023404(1-15)$.

[13] Tolstikhin O.I., Morishita T., Madsen L.B. // Phys. Rev. A. 2011. V. 84. P. 053423 $(1-17)$.

[14] Головинский П.А., Дробышев А.А. // ЖЭТФ. 2014. Т. 145. В. 6. С. 984-990.

[15] Golovinski P.A., Drobyshev A.A. // JEMAA. 2014. V. 6. P. 8-14.

[16] Есеев М.К., Матвеев В.И., Юлкова В.М. // ЖТФ. 2012. Т. 82. В. 11. C. $130-132$.

[17] Demkov Yu.N., Subramanian P. // Sov. Phys. JETP. 1970. V. 30. N 2. P. 381-383.

[18] Dalidchik F.I., Slonim V.Z. // Sov. Phys. JETP. 1976. V. 43. N 1. P. 25-31.

[19] Smirnov B.M., Firsov O.B. // Sov. Phys. JETP. 1965. V. 20. N 1. P. 156-160.

[20] Борзунов С.В., Манаков Н.Л., Старас А.Ф., Фролов М.В. // ЖЭТФ. 2011. T. 139. B. 5. C. $835-855$.

[21] Choi C.-H., Kim C.-J. // Nanotechnology. 2006. V. 17 (21). P. 5326-5333.

[22] Vermal P., Gautam S., Pal S. et al. // Defence Sci. J. 2008. V. 58 (5). P. 650-654.

[23] Chang C.-C., Kuo H.-S., Hwang I.-S., Tsong T.T. // Nanotechnology. 2009. V. 20 (11). P. 115401 (6 p.).

[24] Straton J.C., Bilyeu T.T., Moon B., Moeck P. // Cryst. Res. Technol. 2014. V. 49 (9). P. $663-680$.

[25] Mándi G., Palotás K. // Phys. Rev. B. 2015. V. 91 (16). P. 165406 (1-12). 